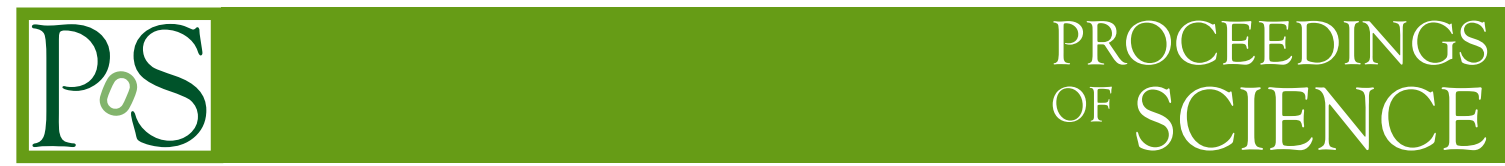

\title{
Forward Physics Results from CMS
}

\author{
Gilvan A. Alves*
}

On behalf of the CMS Collaboration

Lafex/Centro Brasileiro de Pesquisas Fisicas

E-mail: gilvan@cern.ch

This note presents some selected forward physics results from the CERN CMS collaboration. Results are presented on the inelastic cross section at $\sqrt{s}=7 \mathrm{TeV}$, measurement of the Underlying Event activity and the study of jet production at large rapidity separation.

36th International Conference on High Energy Physics,

July 4-11, 2012

Melbourne, Australia

${ }^{*}$ Speaker. 


\section{Introduction}

In hadron collisions, the total cross section includes the sum of elastic, diffractive and hard interactions. The hard interaction itself can be divided in two components: the hard scattering process in which partons are emitted at high transverse momentum with respect to the incoming beams, and therefore can be described in perturbative QCD, and a non-perturbative soft component usually modeled by Monte Carlo event generators. An accurate understanding of the soft component is important for precision measurements of many physics processes to be studied at the LHC.

\section{Inelastic Cross Section}

The cross sections of hadronic collisions are important fundamental quantities in high energy particle, nuclear and cosmic-rays physics and have been studied in the last 60 years in experiments covering many orders of magnitude in center-of-mass energies. The pp total inelastic cross section can not be calculated from perturbative quantum chromodynamics (QCD) but various phenomenological approaches based on fundamental quantum mechanics relations (unitarity, analyticity, and factorisation) exist that can describe the experimental results. We present a measurement of the inelastic proton-proton cross section at $\sqrt{s}=7 \mathrm{TeV}$ with the CMS detector at the LHC[1]. The data were collected in early 2010 during the commissioning phase of the LHC operation with $3.5 \mathrm{TeV}$ beams, corresponding to an integrated luminosity of $2.78 \mathrm{mb}^{-1}$. The events are collected using three triggers: (i) a coincidence trigger that requires the presence of two colliding bunches, used to select an unbiased sample of pp events, (ii) a single-bunch trigger, requiring the presence of just one unpaired bunch, used to estimate beam-induced backgrounds, and (iii) a random 'empty' trigger, requiring absence of both beams, which is used to estimate detector noise. All these triggers are formed from information provided by the beam pick-up detectors. The analysis uses the Hadron Forward Calorimeters (HF) of the CMS apparatus, which are sensitive to most of the inelastic cross section except the fraction coming from diffractive processes in which the dissociated systems have small invariant mass, $M_{X}$. The analysis is based on counting the number of pp collisions with HF energy $E_{H F}>5 \mathrm{GeV}$ in either of the two HF calorimeters. The cross section is evaluated in terms of the variable $\xi=\left(M_{X} c^{2}\right)^{2} / s$, which is defined through MC studies. In single-diffractive events, $\xi$ corresponds to the fraction of momentum lost by the proton in a collision. The number of detected inelastic collisions $\left(N_{\text {inel }}\right)$ contained in the total number of coincident inelastic events $\left(N_{\text {coinc }}\right)$ is obtained as follows:

$$
N_{\text {inel }}=N_{\text {coinc }}\left[\left(F_{\text {coinc }}-F_{\text {empty }}\right)+F_{\text {empty }}\left(F_{\text {coinc }}-F_{\text {empty }}\right)\right]
$$

where $F_{\text {empty }}$ and $F_{\text {coinc }}$ correspond to the fractions of empty and coincidence triggers with $E_{H F}>5$ $\mathrm{GeV}$. The term $N_{\text {coinc }} F_{\text {empty }}\left(F_{\text {coinc }}-F_{\text {empty }}\right)$ represents the number of true collisions in $N_{\text {coinc }} F_{\text {empty }}$ events. The value of $N_{\text {inel }}$ has to be corrected for event pileup, i.e. the possibility that more than one collision with EHF $>5 \mathrm{GeV}$ occurs in the same trigger, but all such collisions are counted as just a single event.

The measured value of the inelastic cross section $\left(\sigma_{\text {inel }}\right)$ for $\xi>5 \times 10^{-6}$ is shown on Figure 1 together with the results for the vertex based analysis (Vtx based) on ref.[1]. The obtained result, 


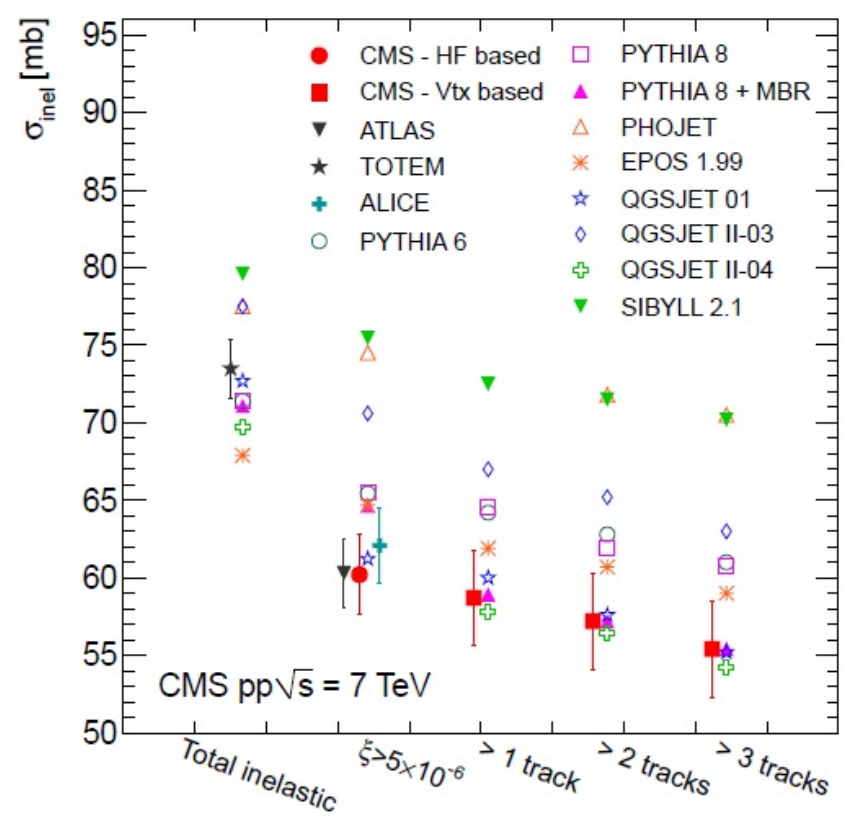

Figure 1: CMS inelastic cross section results compared to predictions from several Monte Carlo models for different criteria, as labelled below the abscissa axis. The MC predictions have an uncertainty of $1 \mathrm{mb}$ (not shown). Other LHC experimental results are also included for comparison.

with statistical and systematic errors is:

$$
\sigma_{\text {inel }}\left(\xi>5 \times 10^{-6}\right)=[60.2 \pm 0.2(\text { stat } .) \pm 1.1(\text { syst. }) \pm 2.4(\text { lum. })] \mathrm{mb}
$$

The sources of systematic uncertainty include the selection efficiency, pileup and contamination corrections.

\section{Measurement of the Underlying Event activity}

The Underlying Event (UE) in a p p collision is everything except the hard scatter- ing. It includes the initial state radiation (ISR), the final state radiation (FSR), the multiple partonic interactions (MPI) and the beam remnants. A good understanding of the UE activity is important in order to extend our knowledge of Quantum Chro- modynamics (QCD) towards the soft limit, for precision measurements of Standard Model processes and searches for new physics. Since the UE is produced by soft or semi-hard interactions, its dynamics can not be fully described by perturbative QCD. As a consequence phenomenological models have to be used whose parameters have to be tuned to data. The measurement of the UE requires a physically motivated separation between the soft and hard components of the collision. In the central region, where there is no separation in pseudorapidity $\eta$ between the hard scattering and the UE, the tradi- tional approach is to divide the phase-space in different $\phi$ regions wrt. the leading $p_{T}$ object. The UE activity is then investigated through the measurement of the energy and particle densities in the so-called towards, transverse and away regions, respectively defined by $|\Delta \phi|<60^{\circ}, 60^{\circ}<|\Delta \phi|<120^{\circ}$ and 

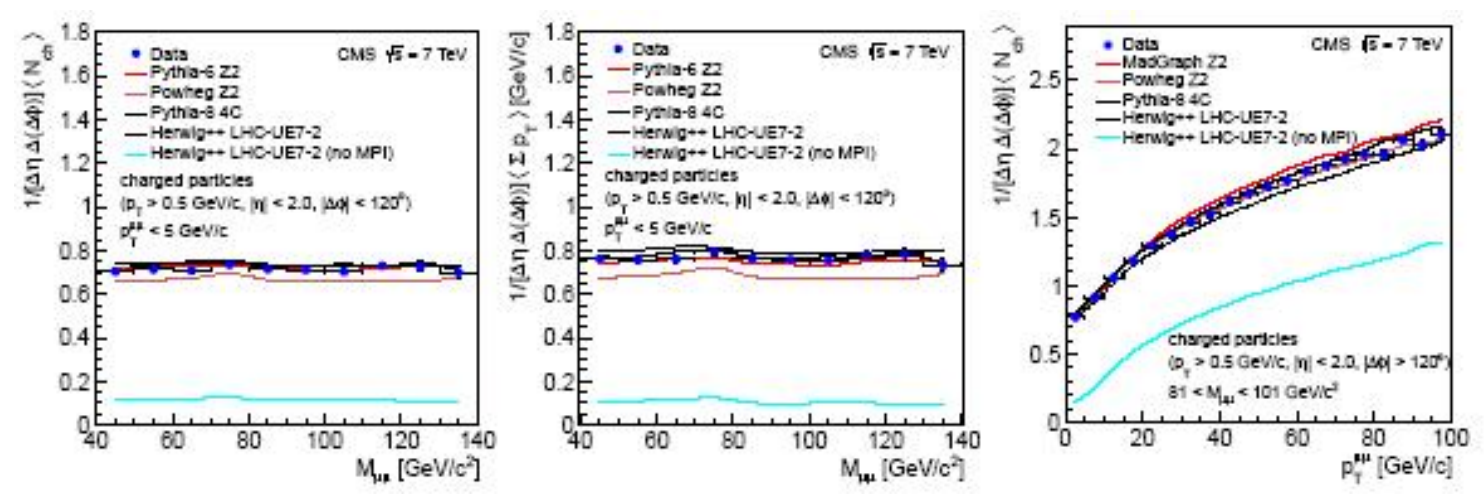

Figure 2: Particle density (left) and energy density (middle) as a function of $M_{\mu \mu}$ in the towards and transverse regions $|\Delta \phi|<120^{\circ}$, with $p_{T}^{\mu \mu}<5 \mathrm{GeV} / \mathrm{c}$. Particle density as a function of $p_{T}^{\mu \mu}$ in the away region (right), with $81<M_{\mu \mu}<101 \mathrm{GeV} / c^{2}$.

$|\Delta \phi|>120^{\circ}$. This approach is followed in the study of the UE activity in the Drell-Yan (DY) process $q \bar{q} \rightarrow \gamma^{*} / Z^{*} \rightarrow \mu^{+} \mu^{-}$[2] which offers a clean separation between the hard scattering and the soft component, is experimentally clean and theoretically well understood. There is furthermore no QCD FSR and limited brehmsstrahlung from the muons, which are required to have $p_{T}>20$ $\mathrm{GeV} / \mathrm{c}$ and $|\eta|<2.4$. The UE activity is determined from the primary charged particles with $p_{T}$ $>0.5 \mathrm{GeV} / \mathrm{c}$ and $|\eta|<2$ and is measured as a function of the dimuon invariant mass $M_{\mu \mu}$ and transverse momentum $p_{T}^{\mu \mu}$. Figure 2 shows the particle density (left) and energy density (middle) at $\sqrt{s}=7 \mathrm{TeV}$ as a function of $M_{\mu \mu}$ in the towards and transverse regions. The condition $p_{T}^{\mu \mu}<5$ $\mathrm{GeV} / \mathrm{c}$ is applied to limit the ISR contribution and the UE is therefore dominated by the MPI, which account for approximately 80presence of a hard scale given by the $\mathrm{Z}$ boson virtuality centers the collision and saturates the MPI. Figure 2 also shows the particle density as function of $p_{T}^{\mu \mu}$ in the away region (right), in the range $81<M_{\mu \mu}<101 \mathrm{GeV} / \mathrm{c} 2$. Since the MPI is saturated at this energy scale, the $p_{T}^{\mu \mu}$ dependence is sensitive to the ISR contribution. The density rises sharply with $p_{T}^{\mu \mu}$ in that region, which is mainly sensitive to the hardest ISR. The slope of the Monte Carlo (MC) prediction is unaffected by the presence of the saturated MPI, showing that the slope is mainly due to the ISR contribution.

In the forward region, the hard scattering and the UE are separated by a large pseudorapidity interval $\Delta \eta$ which makes the phase-space subdivision in $\phi$ unnecessary and offers the possibility to study the $\phi$ dependence of the UE activity. In this approach, the UE activity is measured in the range $-6.6<\eta<-5.2$ by comparing the energy density of two classes of events[3]. Inclusive events, where the energy density is not much affected by MPI are compared to events with a hard scale, in which MPI strongly affect the energy density. The hard scale is defined by the $p_{T}$ of the central leading charged particle jet with $p_{T}>1 \mathrm{GeV} / \mathrm{c}$ and $|\eta|<2$. The energy density in the hard sample is normalized to the inclusive one, making the results independent of calibration and minimizing the systematic uncertainties. Figure 3 shows the ratio of the energy density in the hard and inclusive samples as function of leading charged particle jet $p_{T}$ at $\sqrt{s}=900 \mathrm{GeV}$ (left), $2.76 \mathrm{TeV}$ (middle) and $7 \mathrm{TeV}$ (right). At $7 \mathrm{TeV}$, the typical UE behaviour shows itself by a fast rise at low $p_{T}$ followed by a plateau for $p_{T}>8 \mathrm{GeV}$. At $900 \mathrm{GeV}$, the energy density in the inclusive sample is bigger 

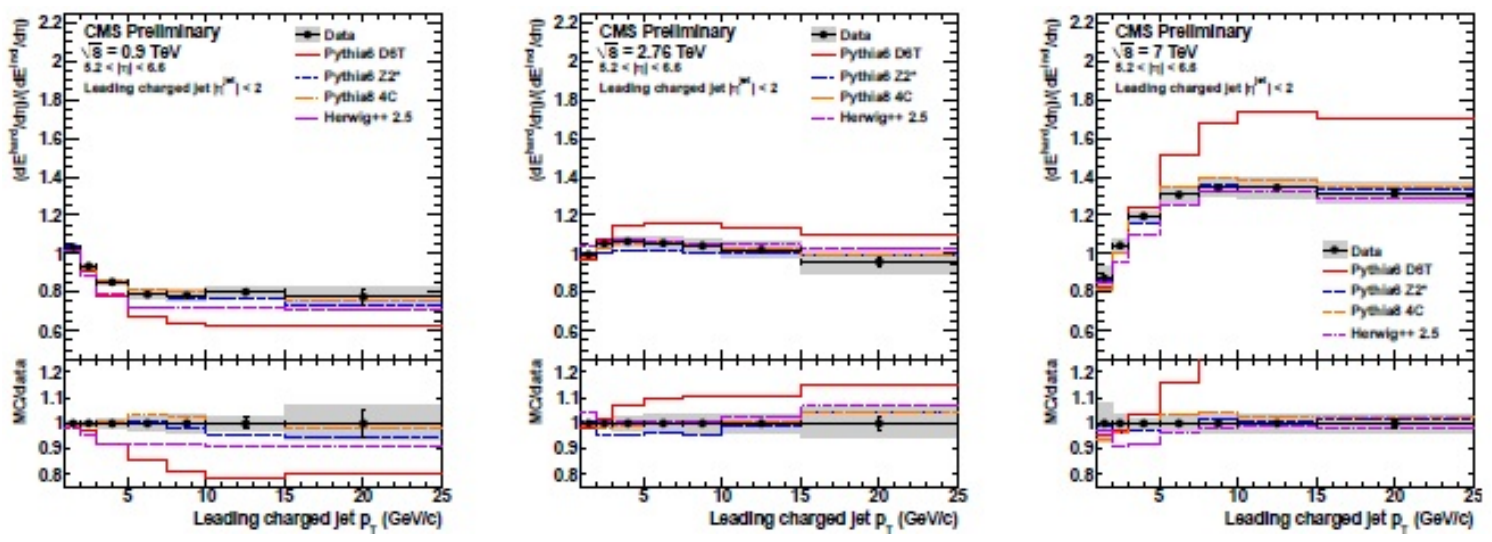

Figure 3: Ratio of the energy density in the hard and inclusive samples as function of leading jet $p_{T}$ at $\sqrt{s}$ $=900 \mathrm{GeV}$ (left), $2.76 \mathrm{TeV}$ (middle) and $7 \mathrm{TeV}$ (right).

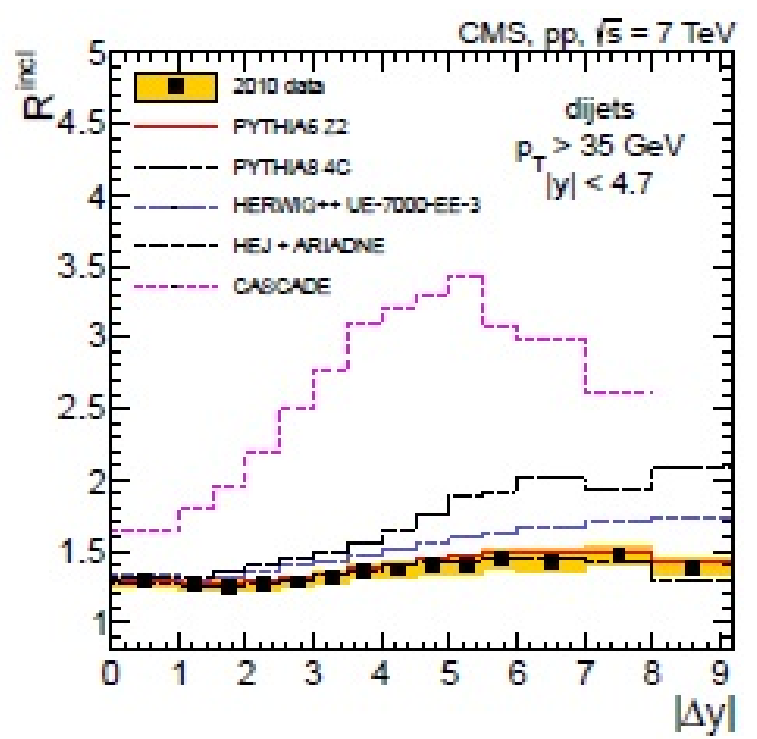

Figure 4: Inclusive to exclusive dijet cross sections ratio as function of the rapidity separation between jets.

than the hard one and the ratio is smaller than unity. At this energy, the proton remnant fragments in the $\eta$ range of the measurement, and the increase of the UE activity in the central region with increasing pT depletes the energy in the hard sample. At $2.76 \mathrm{TeV}$ one observes a much reduced increase of the energy ratio and the activities in both samples are close to each other. The data are well described by PYTHIA6 tunes fitted to LHC data, while the pre-LHC tune PYTHIA6 D6T predicts too much MPI.

\section{Jet production at large rapidity separation}

Dijet production is studied as function of the rapidity separation $\Delta y$ between the jets[4], for jets with $p_{T}>35 \mathrm{GeV}$ and $|y|<4.7$. Two classes of events are considered: inclusive events, with 
at least one pair of jets, and exclusive events, with exactly one pair of jets. The ratio $R_{\text {incl }}$ of the cross section of all pairwise combinations of jets from the inclusive sample to the exclusive dijet cross section is presented on Figure4 as a function of the rapidity difference between the jets $|\Delta y|$. This observable is expected to be sensitive to effects beyond collinear factorization, as the radiation probability increases with $|\Delta y|$ rather than with $p_{T}$. The predictions of the MC event generators PYTHIA6 and PYTHIA8 agree with the measurements, while the HERWIG++ predictions exhibit a more pronounced rise with $|\Delta y|$. The BFKL-motivated generators CASCADE and HEJ+ARIADNE predict for these ratios a significantly stronger rise than observed.

\section{References}

[1] S. Chatrchyan et al. [CMS Collaboration], 'Measurement of the inelastic proton-proton cross section at $\sqrt{s}=7 \mathrm{TeV}$, arXiv:1210.6718.

[2] S. Chatrchyan et al. [CMS Collaboration], 'Measurement of the underlying event in the Drell-Yan process in proton-proton collisions at $\sqrt{s}=7 \mathrm{TeV}$ ', arXiv:1204.1411.

[3] 'Study of the underlying event at forward rapidity in pp collisions at the LHC', CMS PAS FWD-11-003.

[4] S. Chatrchyan et al. [CMS Collaboration], 'Ratios of dijet production cross sec- tions as a function of the absolute difference in rapidity between jets in proton- proton collisions at $\sqrt{s}=7 \mathrm{TeV}$, arXiv:1204.0696. 